\title{
Farrenkopf, Michael; Meyer, Torsten (Hrsg.) (2020): Authentizität und industriekulturelles Erbe. Zugänge und Beispiele
}

\author{
Achim Prossek
}

Eingegangen: 6. August 2021 - Angenommen: 14. September 2021 - Online veröffentlicht: 7. Oktober 2021

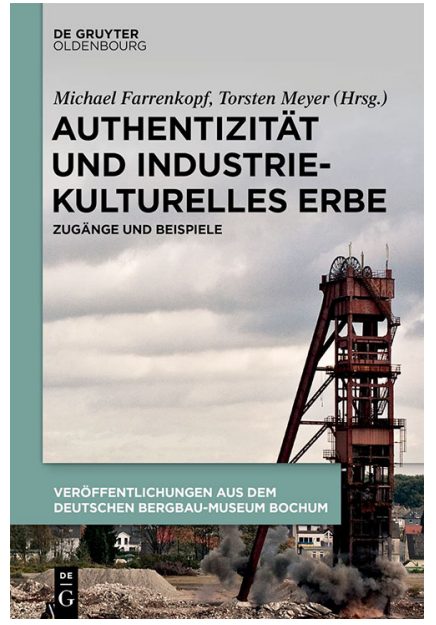

Industriekulturelles Erbe ist ein wichtiges Element der Stadt- und Regionalentwicklung. Es tritt als materielle Hinterlassenschaft auf sowie als sozialer Prozess, als Bedeutungszuschreibung oder als gelebtes, immaterielles Erbe. Authentizität spielt dabei sowohl bei der Unterschutzstellung als auch bei der Restaurierung und dem Umbau wie auch später beim Erleben der Orte eine große Rolle. Für das UNESCO-Welterbe ist sie ein zentrales Kriterium, für die Vermarktung und den Konsum (Tourismus) ebenso.

Dr. Achim Prossek, Kulmer Straße 16, 10783 Berlin, Deutschland achim.prossek@fu-berlin.de

\footnotetext{
(c) (1) 2021 Prossek; licensee oekom verlag. This Open Access article is published under a Creative Commons Attribution 4.0 International License.
}

Damit deuten sich schon unterschiedliche Definitionen und Konflikte an. Ihnen widmet sich der vorliegende Band in interdisziplinärer Perspektive.

Das Buch geht auf die gleichnamige Tagung im April 2017 in Freiberg zurück. Es ist in zwei Abschnitte unterteilt: „Methoden und Perspektiven“ mit sechs sowie „Aspekte und Ausprägungen" mit zehn Beiträgen. Die thematischen Zugänge reichen von der Oral History, Museumspraxis, (Bau-)Denkmalpflege, Fotografie bis zu Praktiken der Urban Explorer, die Fallbeispiele behandeln Regionen wie den amerikanischen Rustbelt, das Ruhrgebiet, das Vogtland, die DDR, den Harz, das Erzgebirge, das rheinisch-westfälische Bergbaurevier sowie Orte in Slowenien, England, der Schweiz, Portugal und Ungarn. Überwiegend wird das montanindustrielle Erbe behandelt.

Die Herausgeber sprechen von einer „Konjunktur“ der Authentizität sowohl in der Praxis als auch zunehmend in der Forschung. Gerade die Industriekultur, welche einzubetten sei in den gesamtgesellschaftlichen Prozess der Transformation von Industrielandschaften, sei bislang aber zu wenig berücksichtigt. Schon die Themen deuten an, dass der Authentizitätsbegriff weit gefasst ist. Wie notwendig und unvermeidlich dies ist, wird beim Lesen schnell deutlich: Während etwa für die Denkmalpflege Kriterien des Ortes und des Materials wichtig sind und auch für Museen der Erhaltungszustand zentral ist, Authentizität also eine objektbezogene Eigenschaft ist, gibt es sie zugleich als soziale Handlung, gibt es Praktikerinnen/Praktiker und Praktiken der Authentisierung. Aus raumplanerischer Sicht sind beide Sichtweisen von Relevanz, weil Planung sowohl mit den materiellen Begebenheiten umgeht, als auch die Raumwirksamkeit sozialer Praktiken berücksichtigen muss. Ausgangspunkt ist dabei oft die Suche nach einer neuen Nutzung für industrielle Hinterlassenschaften, oft unter Berücksich- 
tigung denkmalpflegerischer Vorgaben. Den Bauten wird identifikatorisches sowie oft auch touristisches Potenzial zugesprochen. In mehreren Beiträgen spielt der Welterbe-Status eine Rolle, sodass Fragen des Authentischen nicht nur materielles, sondern auch das immaterielle Kulturerbe betreffen, etwa die Lebenswelt in Arbeitersiedlungen oder die Pflege bergbaulicher Traditionen.

Die Beiträge zur Kolorierung von Fotos und zum Hobby des Urban Exploring, das ist das Fotografieren sogenannter Lost Places, also verlassener Orte, wirken zunächst vielleicht für Planerinnen und Planer weniger relevant, markieren aber im weiteren Sinne das Feld, indem sie populäre Umgangsweisen aufzeigen. Auch sie verhandeln die für die Denkmalpflege, Museologen und Touristikfachleute zentrale Frage, welcher Ortszustand als authentisch anzusehen ist, denn auch Zerstörungen und Nachnutzungen sind ja echt und damit zu einem bestimmten Zeitpunkt authentisch. Zumeist wird aber auf einen Ursprungszustand rekurriert. Authentizität ist mithin immer eine Setzung, und Denkmalschutz und Nutzerinnen/Nutzer der Bauten nehmen diese durchaus verschieden vor. Es wird daher zu Recht fallweise von einer Inszenierung des Authentischen gesprochen. Dass der Begriff nicht auf Originalartefakte zu beschränken ist, zeigt sich am Erfolg von Rekonstruktionen, die gleichfalls oft als authentisch wahrgenommen werden.

Mit dem Ruhrgebiet befassen sich mehrere Beiträge, von der Schaffung einer „Kulturlandschaft neuen Typs“ durch die Internationale Bauausstellung Emscher Park, ihrem geschichtskulturellen Wirken und der Route der Industriekultur als Erinnerungsort wie Tourismusziel. Das industriekulturelle Erbe fungiert als regionale Klammer, es definiert Image und Identität der Region maßgeblich. Deutlich schwieriger ist dies im Harz und auch im Vogtland. Im Harz erstreckt sich das Welterbe (Erzbergwerk Rammelsberg, Goslarer Altstadt, Wasserwirtschaft) räumlich weit. Dies sowie seine Vielgestaltigkeit verhindern Identifikation und Wahrnehmung. Im Vogtland gestaltet es sich als schwierig, die jahrhundertealte reiche Geschichte der Textilindustrie zu erinnern. Dies hat strukturelle und mentale Gründe: Von den zahlreichen Kleinunternehmen ist oft nichts überliefert, die Bauten sind oft nicht markant. Und sächsische Identität speist sich vornehmlich aus Hochkultur oder anderen Industrien (etwa Automobilbau). An den wenigen baulichen Hinterlassenschaften, die als authentische Orte deklariert werden, entzündet sich folglich eine Kontroverse darüber, ob die touristische oder wirtschaftliche Nachnutzung erfolgsversprechender ist.
Lehrreich sind die verschiedenen Beispiele aus der denkmalpflegerischen Praxis, welche aufzeigen, wie situativ begründet unterschiedliche, gleichwohl jeweils authentische Lösungen für den Umgang mit den Denkmälern gefunden wurden. Authentizität beruht meistens auf Kompromissen, im Detail wie im Ganzen, zumeist zwischen Denkmalschutz und Nachnutzungsansprüchen.

Die Beiträge in diesem Band verdeutlichen am Beispiel der Industriekultur, welch hohen Wert Authentizität im Umgang mit dem kulturellen Erbe innehat, als Objektgehalt wie als Zuschreibung oder als Praxis der Authentifizierung. Sie zeigen unterschiedliche Positionen wie Ansprüche auf, von institutionellen Akteuren bis zu losen Zusammenschlüssen von Privatpersonen. Für Planerinnen und Planer ist wichtig, diese Vielfalt zu kennen, aus ihr ergeben sich Herausforderungen wie Chancen für die räumliche Entwicklung. Erkennen und Aushalten von Ambiguität sind, das legen die Beiträge explizit und implizit nahe, beinahe unausweichlich. Bei wenigen wirkt das Thema wie angefügt. Aber auch die dann umfangreicheren Chronologien oder Fallbeispielerläuterungen sind für den Leser und die Leserin lehrreich. Ebenfalls wenigen Beiträgen hätte eine umfangreichere Herleitung der Authentizität gutgetan, um den aufblitzenden Verdacht der Zuschreibung durch den Forschenden selbst zu ersticken. Aber im Kern argumentieren alle überzeugend. Die unterschiedlichen Zugänge und der Praxis- und Ortsbezug machen die Lektüre auch für Beschäftigte in und Interessierte an Raumplanung und Raumwissenschaft lehrreich und anregend.

Während die Denkmalpflege auf eine jahrzehntelange Erfahrung zurückgreifen kann und auch Tourismus und Marketing ihre Strategien kennen, ist das Thema Authentizität aus der Forschungsperspektive noch offener. Das immaterielle Erbe ist zwar im Fokus der Forschung, der Charakter und die Funktion von Authentizität darin jedoch wohl nicht weniger schwer bestimmbar als bei Baudenkmälern. Der Verweis auf ein Gefühl von gelebter Authentizität als Legitimation jedenfalls scheint nicht ausreichend, blickt man etwa auf den unterschiedlichen Ernst, mit dem bergbauliche Tradition im Ruhrgebiet und im Erzgebirge gelebt wird.

\section{Vollständige bibliographische Angaben des rezensierten Werkes:}

Farrenkopf, Michael; Meyer, Torsten (Hrsg.) (2020): Authentizität und industriekulturelles Erbe. Zugänge und Beispiele. Berlin: De Gruyter Oldenbourg. = Veröffentlichungen aus dem Deutschen Bergbaumuseum Bochum 238. 7 Tabellen, 107 Abbildungen, 396 Seiten. 